\title{
Un gène inducteur de la différenciation de l'œil conservé entre les insectes et les mammifères
}

L'importance, chez les mammifères, du gène pax- 6 dans la différenciation de l'œil est attestée par les deux autres noms qu'il porte du fait du phénotype associé à son inactivation: small eye, chez la souris, et $A N I-$ $R I D I A$, chez l'homme $\left(m / s n^{\circ} 2\right.$, vol. 8, p. 181). La mutation hétérozygote de ces gènes entraîne diverses anomalies de l'œil alors que, chez la souris, une inactivation homozygote est associée à une absence d'œil. En 1993, un étudiant doctoral du laboratoire de Walter Gehring, à Bâle (Suisse), clona le gène eyeless de Drosophila melanogaster dont l'inactivation entraîne diverses anomalies de l'œil, jusqu'à leur absence. Eyeless se révéla être un gène pax, homologue des gènes small eye et ANIRI$D I A$, avec $94 \%$ de conservation de séquences au niveau du domaine paired et $90 \%$ de conservation au niveau du domaine homeo, deux motifs protéiques impliqués dans la liaison aux cibles d'ADN $\left(\mathrm{m} / \mathrm{s} n^{\circ} 10\right.$, vol. 10, p. 1058). Cette conservation, des insectes aux mammifères, d'un gène intervenant dans la différenciation de l'œil était en elle-même très surprenante puisque les yeux sont notoirement différents chez les animaux de ces deux classes. En fait, les différences considérables de structure des yeux laissaient à penser que l'œil avait été créé indépendamment plusieurs fois au cours de l'évolution, sans lien, par exemple, entre les phénomènes aboutissant à l'œil des insectes et à celui des mammifères [1]. Or, la conservation d'un même gène impliqué si directement dans la différenciation de l'œil chez la drosophile, la souris et l'homme indique qu'il provient d'un ancêtre

y a plus de 500 millions d'années. Walter Gehring s'est alors posé la question de la place d'eyeless dans la hiérarchie des gènes contrôlant la différenciation d'un œil. En effet, un phénomène de différenciation comporte toujours une cascade d'événements dont les gènes de contrôle peuvent être définis par leur hiérarchie, c'est-à-dire par leur position dans la cascade et par leur pouvoir de contrôler les gènes d'un niveau inférieur dans la hiérarchie. Un gène nécessaire et suffisant pour déclencher, à lui tout seul, la totalité du processus de différenciation sera appelé le gène de contrôle principal (master control gene). Chez les mammifères, les différenciateurs myogéniques de la famille $M y o-D$, capables d'entraîner, lorsqu'ils sont surexprimés, la transdifférenciation de différents types cellulaires en myotubes, sont ainsi considérés comme étant très élevés dans la hiérarchie $(\mathrm{m} / \mathrm{s}$ $n^{\circ} 4$, vol. 4, p. 255). L'existence de gènes de contrôle principaux des voies de différenciation chez la drosophile était suggérée par des expériences anciennes de transdifférenciation des disques imaginaux. Ces disques embryonnaires sont à l'origine des structures adultes, comme les ailes, les yeux, les pattes, etc. Lorsqu'un disque imaginal est prélevé et cultivé, il se différencie presque toujours dans le respect de son identité. Dans certains cas, cependant, se produit un phénomène de transdifférenciation, aussi appelé transdétermination, au cours duquel un disque d'une identité particulière, par exemple destiné à donner une aile, se différencie en un autre organe, par exemple un œil. L'activation accidentelle d'un gène de contrôle principal, normalement réprimé dans le disque considéré, peut expliquer un tel phénomène. Pour tester l'hypothèse selon laquelle le gène eyeless pouvait se comporter comme un gène de contrôle principal, Halder et al., de l'équipe de Walter Gehring, ont étudié les effets d'une expression ectopique du gène eyeless [2]. Celle-ci a été obtenue en utilisant la même méthode que celle résumée dans médecine/sciences par J.F. Ferveur ( $m / s n^{\circ} 5$, vol. 11, p. ???). En résumé, les auteurs ont utilisé une collection de lignées de drosophiles transgéniques pour le gène GAL-4 dépourvu des séquences nécessaires à son expression. De ce fait, l'expression de $G A L-4$ ne peut se faire que sous le contrôle du enhancer d'un gène cellulaire dans lequel, ou à proximité duquel, le transgène est intégré. Par ailleurs, des souris transgéniques possédant un transgène eyeless ou lac- $Z$ placé sous le contrôle des éléments UAS (upstream activating sequence) sont utilisées. Ces transgènes ne sont activés que lorsque la protéine GAL-4 se fixe sur les séquences UAS. Un croisement des souris $G A L-4$ et lac $Z$ permet, grâce à la révélation histochimique de l'activité $\beta$-galactosidase, produit du gène lac-Z, de détecter dans quelles cellules est exprimé l'activateur GAL-4; dans ces cellules seulement, le transgène lac- $Z$ sera stimulé, permettant la coloration histochimique pour la $\beta$-galactosidase. Le remplacement de la lignée lac-Z par la lignée eyeless permet alors d'observer les conséquences de l'expression ectopique de la protéine Eyeless dans les cellules considérées. Le résultat est spectaculaire: cette synthèse ectopique de la protéine 
Eyeless provoque l'apparition ectopique d'yeux parfaitement formés, sensibles à la lumière et fonctionnels. Ces yeux peuvent se trouver au niveau d'une antenne, d'une patte, d'une aile, etc. Jusqu'à 14 yeux ont pu ainsi être obtenus chez les animaux. L'homologie fonctionnelle entre eyeless de drosophile et small eye de souris est démontrée par l'aptitude de ce dernier gène d'induire, comme eyeless, le développement d'yeux en des sites ectopiques. Par conséquent, la grande hétérogénéité de la structure des yeux ne semble pas due à l'apparition indépendante d'organes visuels plusieurs fois au cours de l'évolution: les résultats de Walter Gehring, résumés ici, et le fait que des homologues du gène eyeless/small eye/ANIRIDIA soient observés chez des calamars et même des planaires, petits vers plats possédant des yeux punctiformes élémentaires, suggèrent qu'a pu exister un ancêtre commun extrêmement ancien possédant une ébauche d'organe visuel d'où sont dérivés tous les yeux actuels. En revanche, au-delà de l'activation de la différenciation de l'œil par l'ancêtre du gène eyeless, des gènes différents placés sous le contrôle du master gene commun ont pu apparaître et régler l'édification de structures oculaires variées.

A.K.

1. Barinaga M. Focusing on the eyeless gene. Science 1995; 267 : 1766-7.

2. Halder G, Callaerts P, Gehring WJ, Induction of ectopic eyes by targeted expression of the eyeless gene in Drosophila. Science 1995; 267 : 1788-92. 